\title{
Research Article \\ Generalized Derivations and Left Ideals in Prime and Semiprime Rings
}

\author{
Basudeb Dhara and Atanu Pattanayak \\ Department of Mathematics, Belda College, Paschim Medinipur, Belda 721424, India \\ Correspondence should be addressed to Basudeb Dhara, basu_dhara@yahoo.com
}

Received 19 May 2011; Accepted 7 July 2011

Academic Editors: V. De Filippis and A. Rapinchuk

Copyright (C) 2011 B. Dhara and A. Pattanayak. This is an open access article distributed under the Creative Commons Attribution License, which permits unrestricted use, distribution, and reproduction in any medium, provided the original work is properly cited.

Let $R$ be an associative ring, $\lambda$ a nonzero left ideal of $R, d: R \rightarrow R$ a derivation and $G: R \rightarrow R$ a generalized derivation. In this paper, we study the following situations in prime and semiprime rings: (1) $G(x \circ y)=a(x y \pm y x) ;(2) G[x, y]=a(x y \pm y x)$; (3) $d(x) \circ d(y)=a(x y \pm y x)$; for all $x, y \in \lambda$ and $a \in\{0,1,-1\}$.

\section{Introduction}

Throughout this paper, let $R$ be an associative ring, $\lambda$ a left ideal of $R, d$ a derivation of $R$ and $G$ a generalized derivation of $R$. For any two elements $x, y \in R,[x, y]$ will denote the commutator element $x y-y x$ and $x \circ y$ denotes $x y+y x$. We use extensively the following basic commutator identities: $[x y, z]=[x, z] y+x[y, z]$ and $[x, y z]=[x, y] z+y[x, z]$. Recall that a ring $R$ is called prime, if for any $a, b \in R, a R b=(0)$ implies that either $a=0$ or $b=0$ and is called semiprime if for any $a \in R, a R a=(0)$ implies $a=0$. An additive mapping $d: R \rightarrow R$ is said to be a derivation of $R$ if for any $x, y \in R, d(x y)=d(x) y+x d(y)$ holds. The generalized derivation of $R$ is defined as an additive mapping $G: R \rightarrow R$ such that $G(x y)=G(x) y+x d(y)$ holds for any $x, y \in R$, where $d$ is a derivation of $R$. So, every derivation is a generalized derivation, but the converse is not true in general. If $d=0$, then we have $G(x y)=G(x) y$ for all $x, y \in R$, which is called a left multiplier mapping of $R$. Thus, generalized derivation generalizes both the concepts, derivation as well as left multiplier mapping of $R$.

In [1], Daif and Bell proved that if $R$ is a semiprime ring with a nonzero ideal $I$ and $d$ is a derivation of $R$ such that $d([x, y])= \pm[x, y]$ for all $x, y \in I$, then $I$ is central ideal. In particular, if $I=R$, then $R$ is commutative. Recently, Quadri et al. [2] have generalized this result replacing derivation $d$ with a generalized derivation in a prime ring $R$. More precisely, they obtained the following result. 
Let $R$ be a prime ring and $I$ a nonzero ideal of $R$. If $R$ admits a generalized derivation $F$ associated with a nonzero derivation d such that any one of the following holds: (i) $F([x, y])=[x, y]$ for all $x, y \in I$; (ii) $F([x, y])=-[x, y]$ for all $x, y \in I$; (iii) $F(x \circ y)=(x \circ y)$ for all $x, y \in I$; (iv) $F(x \circ y)=-(x \circ y)$ for all $x, y \in I$; then $R$ is commutative.

Recently in [3], the first author of this paper has studied all the results of [2] in semiprime ring. In the present paper, our aim is to discuss similar identities in a left sided ideal of a semiprime rings.

\section{Main Results}

Theorem 2.1. Let $R$ be a semiprime ring and $\lambda$ a nonzero left ideal of $R$. If $G$ is a generalized derivation of $R$ associated with a derivation $d$ of $R$ such that $G(x \circ y)=a(x \pm y)$ for all $x, y \in \lambda$, where $a \in\{0,1,-1\}$, then $[\lambda, \lambda] d(\lambda)=0$.

Proof. If $G(\lambda)=0$, then for any $x, y \in \lambda$,

$$
0=G(x y)=G(x) y+x d(y)=x d(y)
$$

that is, $\lambda d(\lambda)=0$. This gives our conclusion. So let $G(\lambda) \neq 0$. Then by our assumption, we have

$$
G(x \circ y)=a(x \pm y)
$$

for all $x, y \in \lambda$. Putting $y=y x, x \in \lambda$, we obtain that $G((x \circ y) x)=a((x \pm y) x)$. Since $G$ is a generalized derivation of $R$, this implies that $G(x \circ y) x+(x \circ y) d(x)=a(x \pm y) x$. This gives by using (2.2) that $(x \circ y) d(x)=0$ for all $x, y, \in \lambda$. Now, we replace $y$ with $z y$, where $z \in \lambda$, and then we get

$$
0=z(x \circ y) d(x)+[x, z] y d(x)=[x, z] y d(x)
$$

for all $x, y, z \in \lambda$. Since $\lambda$ is a left ideal, it follows that $[x, z] \operatorname{Ryd}(x)=0$ for all $x, y, z \in \lambda$. Since $R$ is semiprime, it must contain a family $\Omega=\left\{P_{\alpha}: \alpha \in \Lambda\right\}$ of prime ideals such that $\bigcap_{\alpha \in \Lambda} P_{\alpha}=\{0\}$. If $P$ is typical member of $\Omega$ and $x \in \lambda$, we have either $[x, \lambda] \subseteq P$ or $\lambda d(x) \subseteq P$. For fixed $P$, the sets $T_{1}=\{x \in \lambda:[x, \lambda] \subseteq P\}$ and $T_{2}=\{x \in \lambda: \lambda d(x) \subseteq P\}$ form two additive subgroups of $\lambda$ such that $T_{1} \cup T_{2}=\lambda$. Therefore, either $T_{1}=\lambda$ or $T_{2}=\lambda$, that is, either $[\lambda, \lambda] \subseteq P$ or $\lambda d(\lambda) \subseteq P$. Both of these two conditions together imply that $[\lambda, \lambda] d(\lambda) \subseteq P$ for any $P \in \Omega$. Therefore, $[\lambda, \lambda] d(\lambda) \subseteq \bigcap_{\alpha \in \Lambda} P_{\alpha}=0$.

Corollary 2.2. Let $R$ be a prime ring and $\lambda$ be a nonzero left ideal of $R$. If $R$ admits a generalized derivation $G$ associated with a derivation $d$ such that $G(x \circ y)=a(x \circ y)$ for all $x, y \in \lambda$, where $a \in\{0,1,-1\}$, then one of the following holds:

(i) $\lambda d(\lambda)=0$;

(ii) $R$ is commutative ring with char $(R)=2$;

(iii) $R$ is commutative ring with char $(R) \neq 2$ and $G(x)=$ ax for all $x \in \lambda$. 
Proof. By Theorem 2.1, we have $[\lambda, \lambda] d(\lambda)=0$. This gives

$$
0=\left[\lambda, \lambda^{2}\right] d(\lambda)=[\lambda, \lambda] \lambda d(\lambda)=[\lambda, \lambda] R \lambda d(\lambda)
$$

Since $R$ is prime, either $[\lambda, \lambda]=0$ or $\lambda d(\lambda)=0$. Now $\lambda d(\lambda)=0$ gives our conclusion (i). So, let $[\lambda, \lambda]=0$ which gives $0=[\lambda, R \lambda]=[\lambda, R] \lambda$ implying $0=[\lambda, R]$. Again, this gives $0=[R \lambda, R]=[R, R] \lambda$. Since left annihilator of a left-sided ideal is zero, $[R, R]=0$, that is, $R$ is commutative. If $\operatorname{char}(R)=2$, we obtain conclusion (ii). So assume that $\operatorname{char}(R) \neq 2$. Then our assumption $G(x \circ y)=a(x \circ y)$ gives $2 G(x y)=2 a(x y)$ for all $x, y \in \lambda$. Since $\operatorname{char}(R) \neq 2$, then $G(x y)=a(x y)$ for all $x, y \in \lambda$. This gives for all $x, y \in \lambda$,

$$
0=G(x y)-a(x y)=G(x) y+x d(y)-a x y=(G(x)-a x) y+x d(y) .
$$

Let $r \in R$. Since $R$ is commutative, $x r \in \lambda$. Put $x=x r$ in last result, we get $0=(G(x)-a x) r y+$ $x r d(y)+x d(r) y=\{(G(x)-a x) y+x d(y)\} r+d(r) y x$. Since $R$ is commutative, using (2.5), it yields $d(R) \lambda^{2}=\{0\}$, implying $d=0$. Then, (2.5) implies $(G(x)-a x) \lambda=0$, which yields $G(x)-a x=0$ for all $x \in \lambda$.

Theorem 2.3. Let $R$ be a semiprime ring, $\lambda$ a nonzero left ideal of $R$ and $G$ a generalized derivation of $R$ associated with a derivation $d$ of $R$. If $G[x, y]=a(x y \pm y x)$ for all $x, y \in \lambda$, then $[\lambda, \lambda] d(\lambda)=0$.

Proof. If $G(\lambda)=0$, then $0=G\left(\lambda^{2}\right)=G(\lambda) \lambda+\lambda d(\lambda)=\lambda d(\lambda)$ and hence, $[\lambda, \lambda] d(\lambda)=0$, which is our conclusion. Assume next that $G(\lambda) \neq 0$. Then by our assumption, we have

$$
G[x, y]=a(x y \pm y x)
$$

for all $x, y \in \lambda$. Put $y=y x$ and get $G([x, y] x)=a(x y \pm y x) x$, that is, $G([x, y]) x+[x, y] d(x)=$ $a(x y \pm y x) x$. Now using (2.6), the above relation yields $[x, y] d(x)=0$ for all $x, y \in \lambda$. Putting $y=z y$, where $z \in \lambda$, we obtain that $[x, z] y d(x)=0$, which is same as (2.3) in Theorem 2.1. By same argument of Theorem 2.1, we can conclude the result here.

Corollary 2.4. Let $R$ be a prime ring and $\lambda$ a nonzero left ideal of $R$. If $G$ is a generalized derivation of $R$ associated with a derivation $d$ of $R$ such that $G[x, y]=a(x y \pm y x)$ for all $x, y \in \lambda$, where $a=\{0,1,-1\}$, then either $R$ is commutative or $\lambda d(\lambda)=0$ and one of the following holds:

(i) $\lambda[\lambda, \lambda]=0$;

(ii) $G(x)=$ Fax for all $x \in \lambda$. In case $G(x)=-$ ax for all $x \in \lambda$, with $a \neq 0$, then $\operatorname{char}(R)=2$.

Proof. By the Theorem 2.3, we may conclude that $[\lambda, \lambda] d(\lambda)=0$. Then by same argument as given in Corollary 2.2, we obtain that either $R$ is commutative or $\lambda d(\lambda)=0$. Let $R$ be noncommutative, then for any $x, y \in \lambda$, we have $G(x y)=G(x) y+x d(y)=G(x) y$, that is, $G$ acts as a left multiplier map on $\lambda$. Then for any $x, y, z \in \lambda$, replacing $y$ with $y z$ in our hypothesis $G[x, y]=a(x y \pm y x)$, we have

$$
G([x, y] z+y[x, z])=a\{(x y \pm y x) z \mp y[x, z]\}
$$


for all $x, y \in \lambda$. Since $G$ acts as a left multiplier map on $\lambda$, this implies

$$
G([x, y]) z+G(y)[x, z]=a(x y \pm y x) z \mp a y[x, z]
$$

By using $G[x, y]=a(x y \pm y x)$, it gives $G(y)[x, z]=\mp a y[x, z]$, that is, $(G(y) \pm a y)[x, z]=0$ for all $x, y \in \lambda$. Replacing $y$ with $y u$, where $u \in \lambda$, we find that $(G(y) \pm a y) u[x, z]=0$, which gives $(G(y) \pm a y) R \lambda[x, z]=0$. Since $R$ is prime, either $\lambda[\lambda, \lambda]=0$ or $G(y)=\mp a y$ for all $y \in \lambda$. When $G(y)=-a y$, our assumption $G[x, y]=a(x y+y x)$ implies $-a[x, y]=a(x y+y x)$ for all $x, y \in \lambda$. This implies $2 a x y=0$, that is, $2 a R \lambda^{2}=0$ implies char $(R)=2$, unless $a=0$.

Theorem 2.5. Let $R$ be a semiprime ring, $\lambda$ a nonzero left ideal of $R$ and $d$ a derivation of $R$. If $d(x) \circ d(y)=a(x y \pm y x)$ for all $x, y \in \lambda$, where $a \in\{0,1,-1\}$, then $\lambda[x, d(x)]_{2}=0$. In case $\lambda=R$ and $R$ is 2-torsion free, $d$ maps $R$ into its center.

Proof. We have for all $x, y \in \lambda$,

$$
d(x) d(y)+d(y) d(x)=a(x y \pm y x)
$$

Putting $y=y x$, we get

$$
d(x)(d(y) x+y d(x))+(d(y) x+y d(x)) d(x)=a(x y \pm y x) x .
$$

Using (2.9), we have

$$
d(x) y d(x)+d(y)[x, d(x)]+y d(x)^{2}=0
$$

Put $y=x y$ in (2.11), and get

$$
d(x) x y d(x)+\{x d(y)+d(x) y\}[x, d(x)]+x y d(x)^{2}=0 .
$$

Left multiplying (2.11) by $x$ and then subtracting from (2.12) yields

$$
[d(x), x] y d(x)+d(x) y[x, d(x)]=0
$$

for all $x, y \in \lambda$. Put $y=d(x) y$ in (2.13), we have

$$
[d(x), x] d(x) y d(x)+d(x)^{2} y[x, d(x)]=0 .
$$

Left multiplying (2.13) by $d(x)$ and then subtracting from (2.14), we get $[x, d(x)]_{2} y d(x)=0$ for all $x, y \in \lambda$. This implies $[x, d(x)]_{2} y[x, d(x)]_{2}=0$ and hence, $y[x, d(x)]_{2} R y[x, d(x)]_{2}=0$ for all $x, y \in \lambda$. Since $R$ is semiprime, $\lambda[x, d(x)]_{2}=0$. In case $\lambda=R,[x, d(x)]_{2}=0$ for all $x \in R$, and then by [4], $d(R) \subseteq Z(R)$. 


\section{References}

[1] M. N. Daif and H. E. Bell, "Remarks on derivations on semiprime rings," International Journal of Mathematics and Mathematical Sciences, vol. 15, no. 1, pp. 205-206, 1992.

[2] M. A. Quadri, M. S. Khan, and N. Rehman, "Generalized derivations and commutativity of prime rings," Indian Journal of Pure and Applied Mathematics, vol. 34, no. 9, pp. 1393-1396, 2003.

[3] B. Dhara, "Remarks on generalized derivations in prime and semiprime rings," International Journal of Mathematics and Mathematical Sciences, vol. 2010, Article ID 646587, 6 pages, 2010.

[4] M. T. Koşan, T.-K. Lee, and Y. Zhou, "Identities with Engel conditions on derivations," to appear in Monatshefte für Mathematik. 


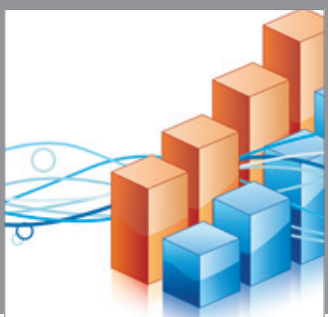

Advances in

Operations Research

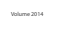

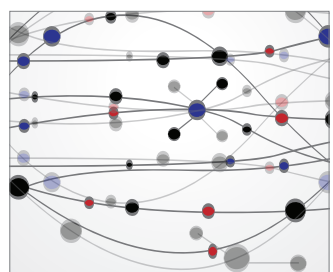

\section{The Scientific} World Journal
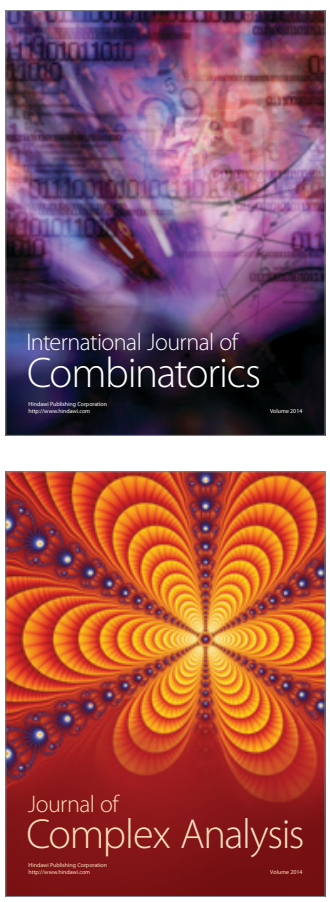

International Journal of

Mathematics and

Mathematical

Sciences
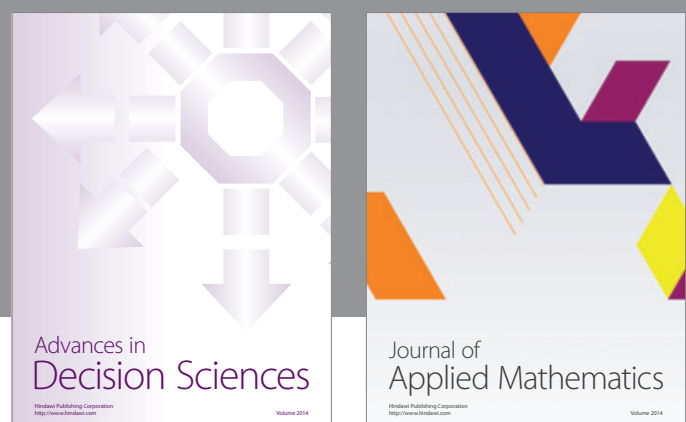

Journal of

Applied Mathematics
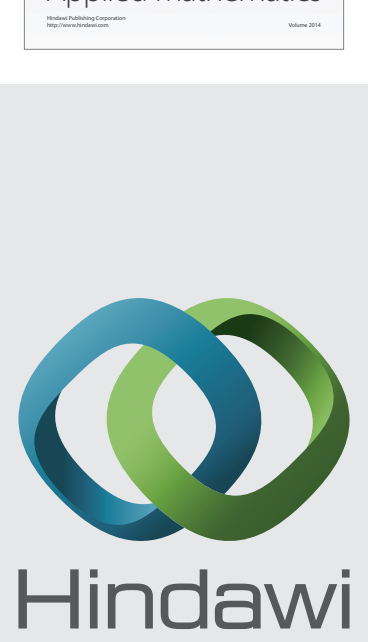

Submit your manuscripts at http://www.hindawi.com
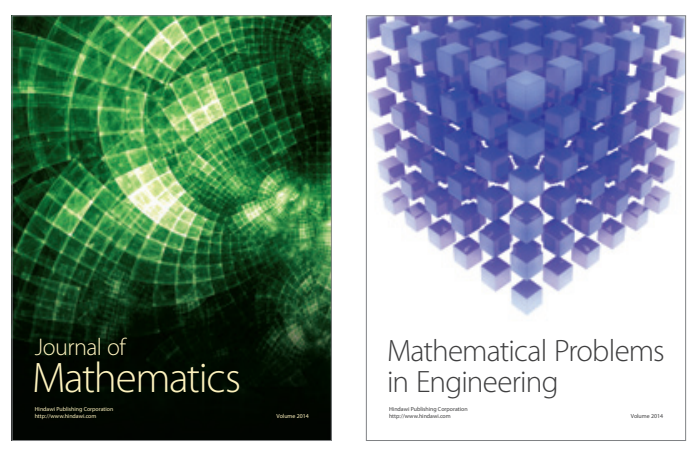

Mathematical Problems in Engineering
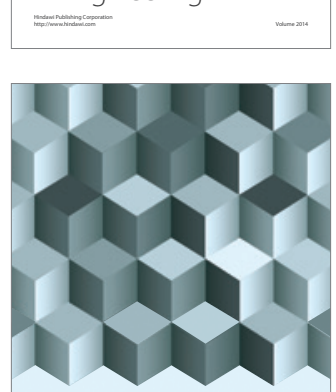

Journal of

Function Spaces
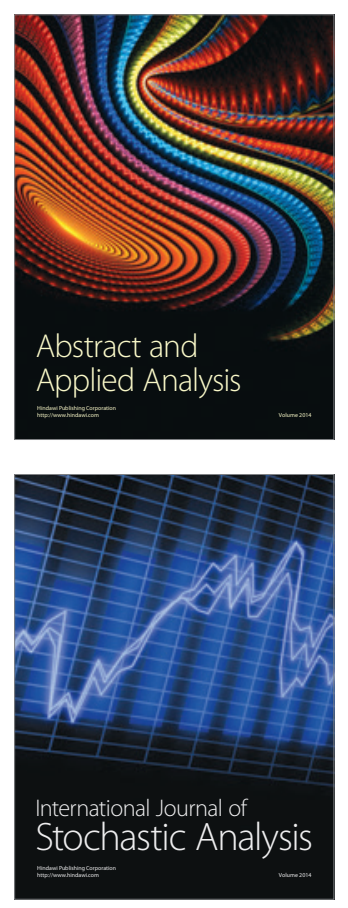

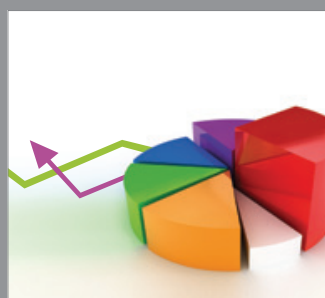

ournal of

Probability and Statistics

Promensencen
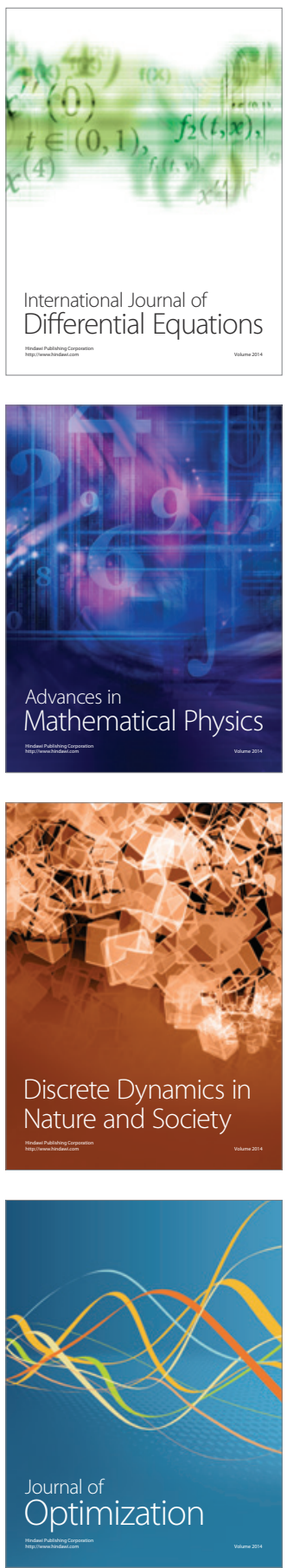